\title{
The effect of directed forgetting on the time to remember
}

\author{
WILLIAM EPSTEIN, LUCINDA WILDER, and LYNN ROBERTSON \\ University of Wisconsin-Madison, Wisconsin 53706
}

\begin{abstract}
A recognition memory task was used to determine whether the search set is reduced by cueing subject to forget a part of the input. Correct recognition reaction time was used to infer the size of the search set. The principal results were that RT on forget trials was faster than RT on remember trials and that RT was fastest when the forget cue was presented at the outset of the trial rather than after the material had been presented. These findings are generally consistent with the hypothesis that selective search is responsible for the enhancement of recall produced by forget instructions in earlier studies.
\end{abstract}

In a variety of experimental paradigms (Bjork, 1973; Epstein, 1972) directing the subject to forget part of the material after it has been presented, enhanced recall of to-be-remembered material relative to recall of the same material in the absence of forget instructions has been reported. Epstein et al. (Epstein, Massaro, \& Wilder, 1972; Epstein \& Wilder, 1972; Shebilske \& Epstein, 1973) have hypothesized that selective search is the mechanism responsible for the effectiveness of forget instructions in the paired-associate probe paradigm. According to the selective search hypothesis, on a test that follows a forget cue, subject restricts the search to the to-be-remembered set of pairs and excludes the to-be-forgotten pairs from consideration. It is this reduction in the size of the search set on forget trials that is responsible for the enhancement of recall. Starting with the premise that the time required to retrieve a pair depends on the size of the search set, the present experiments sought to provide evidence that forget instructions actually do reduce the size of the search set.

Evidence that cueing can define the search set and affect search time is already available in the studies reported by DeRosa et al. (Crain \& DeRosa, 1974; DeRosa, 1969; Kaminsky \& DeRosa, 1972). These studies have shown that, on a Sternberg-type recognition memory task, the size of the search set, as distinguished from the memory set, can be controlled by postinput cueing and that RT for correct recognition is a linear increasing function of the size of the set that subject has been implicitly instructed to search.

An experiment that is immediately related to our concerns has been reported by Howard (Note 1). She presented two sets of two pictorial paired associates on each trial followed by a probe recognition test in which subject responded "yes" if the test pair was identical with one of the list pairs and "no" otherwise. Two types

This research was supported by Grant MH 16390 from the U.S. Department of Health, Education, and Welfare.

Requests for reprints should be sent to William Epstein, Department of Psychology, University of Wisconsin, Madison, Wisconsin 53706 . of incorrect pairings were presented on the test, an old stimulus paired with a wrong response from the same set as the correct response, and an old stimulus paired with a response from the set which did not contain the correct response. Each trial was accompanied by one of three cues. A "remember" cue required subject to remember all four pairs since any of the four might be tested. A "forget during" cue presented at the outset of the trial informed subject that only one of the two sets would be tested. A "forget after" cue presented following the last pair informed subject that only one of the sets should be tested. Reaction times were measured from the onset of each test pair. Howard found that RT was faster on forget during and forget after trials than on remember trials. This finding is consistent with the claim that forget cues can act to reduce the size of the search set.

The present experiments adapted the task devised by Howard (1973). Our principal aim was to determine whether similar effects of forget instructions would be observed with verbal materials. In addition, procedural revisions were introduced to afford closer correspondence with the format of our previous paired-associate probe studies. For example, on the forget during trials Howard omitted the stimuli for the to-be-forgotten pairs, while the present procedure presented four complete pairs on each trial. Howard provided feedback after each response, while we did not include feedback. These differences, as well as others, necessarily limit comparisons between the results of the present experiments and the results reported by Howard to general trends.

\section{EXPERIMENT I}

\section{Method}

Subjects. Thirty-four subjects drawn from the University of Wisconsin subject pool participated in the experiment. An additional 11 subjects were tested but discarded because they made too many errors.

Materials and apparatus. Every subject viewed 96 four-pair lists, one list at a time, in four blocks of 24 lists. Each list 
Table 1

Mean Correct RT (Milliseconds) and Error Proportion for Cue and Solution Types in Experiments I and II

\begin{tabular}{lcccccc}
\hline & & \multicolumn{3}{c}{$\begin{array}{c}\text { Experiment I } \\
\text { Cue Type }\end{array}$} & & \multicolumn{2}{c}{$\begin{array}{c}\text { Experiment II } \\
\text { Cue Type }\end{array}$} \\
\cline { 3 - 6 } Solution Type & Remember & $\begin{array}{c}\text { Forget } \\
\text { After }\end{array}$ & $\begin{array}{c}\text { Forget } \\
\text { Before }\end{array}$ & Remember & $\begin{array}{c}\text { Forget } \\
\text { After }\end{array}$ \\
\hline Yes & 1838 & 1802 & 1464 & 1964 & 1812 & 1502 \\
& $(.23)$ & $(.17)$ & $(.08)$ & $(.20)$ & $(.16)$ & $(.10)$ \\
No-Same & 2149 & 2024 & 1739 & 2113 & 2002 & 1812 \\
& $(.20)$ & $(.127)$ & $(.15)$ & $(.24)$ & $(.27)$ & $(.15)$ \\
No-Different & 2020 & 1843 & 1616 & 2124 & 1883 & 1671 \\
& $(.25)$ & $(.11)$ & $(.06)$ & $(.26)$ & $(.08)$ \\
\hline
\end{tabular}

consisted of four CVC syllables as stimuli and four nouns as responses. The same four syllables, BOK, MIV, WOB, GUK were repeated in each list; however, none of the nouns were repeated within a block. In each list the nouns serving for the first two pairs were selected from the same taxonomic category (Battig \& Montague, 1969), and the nouns serving as responses in the second set were drawn from a different category. Twenty-four different categories were used in forming the 96 lists. With the exception of the instructions, all material was presented visually on lightemitting diode displays. Presentation rates were controlled by an Automated Data Systems 1800 system. The control and recording equipment was housed in one room and the subjects in another. Each subject's desk top was equipped with a panel containing two springtoaded pushbuttons, marked "yes" and "no." In the group of subjects, the right hand was assigned equally often to the "yes" and "no" buttons. When not responding, subject rested the index fingers on the buttons. Choice of response and reaction time in msec was recorded automatically on Teletype tape.

Design. There were no between-subject variables. Cue (forget before, forget after, remember), solution type (yes, no-same, no-different), and serial position of the tested pair were all within-subject variables. Several different random orders of assignment of pairs to cues and solution types were employed.

Procedure. Subjects were tested in small groups not exceeding four subjects. Each subject began by listening to a detailed description of the task and then running through 24 practice trials. The description included information about the composition of the lists, the meaning of the cues, and the requirements of the task: "After the fourth pair a test pair will appear which contains one of the syllables and one of the words that were presented on the trial ... Push the button labeled "yes" if the pair presented on the test trial is identical to the pair presented earlier on the same trial ... Push the button "no" if the word presented is not the word that was paired with the nonsense syllable ... Respond as quickly as you can without making errors."

Each trial began with a 1-sec ready signal. Then, on a forget before trial the forget cue, "first" or "second" appeared for $.5 \mathrm{sec}$ informing subject that the test would involve only the designated set. The first set of two CVC-noun pairs followed, each pair presented individually for $4 \mathrm{sec}$. Then a row of asterisks for $.5 \mathrm{sec}$ to help distinguish the sets, followed by the second set of two pairs. After the fourth pair there was a $.5 \mathrm{sec}$ interval which was blank on the forget before trials and contained the cue on the other trials. On the forget after trials, the cue "first" or "second appeared for $.5 \mathrm{sec}$; on remember trials the cue "either" appeared. The cue "first" or "second" directed subject to forget the excluded set because the test would probe only the designated set; the cue "either" directed subject to remember all four pairs because the test would probe either of the two sets. Finally, the test pair appeared for 5 sec. Only the first response was recorded. Any response made after the $5 \mathrm{sec}$ elapsed was recorded as an error. There was a 2 -sec interval between offset of the probe pair and the next ready signal. In each block of 24 trials, 12 probes were identical with one of the pairs, calling for a "yes" response, and 12 probes were different, 6 "no-same" and 6 "no-different." On "no-same" probes the stimulus was paired with the other response from the same set; on "no-different" probes the stimulus was paired with a response from the other set. For example, in the list: GUK-MILK, MIV-BEER****WOB-TULIP, BOK-ROSE, the test GUK-BEER is a "no-same" probe and GUK-TULIP is a "no-different" probe.

\section{Results}

The primary results of the experiment are given in Table 1. Mean reaction time for correct recognition is shown as a function of cue type and solution type. Both variables affected RT. Responses were fastest on forget before trials, slowest on remember trials and intermediate on forget after trials. Responses were fastest to "yes" probes, slowest to "no-same" probes, and intermediate to "no-different" probes. These observations were confirmed by analysis of variance of correct RTs. The main effects of cue, $F(2,66)=37.94$ and solution type, $F(2,66)=21.81$, were significant. There was also a significant effect associated with the position of the probed pair, $\mathrm{F}(3,81)=9.17, \mathrm{p}<.01$. Figure 1 shows the effect to be the conventional bowed serial position curve. No interactions were significant.

Examination of the individual data showed that $73 \%$ of the subjects had shorter RTs on forget after than on remember trials and $88 \%$ had faster RTs on forget before than forget after trials. In both cases, the distribution differs significantly from chance.

Error rate was directly related to the magnitude of correct RT. Table 1 shows that error proportions were highest on remember trials, intermediate on forget after trials, and lowest on forget before trials.

\section{EXPERIMENT II}

Although forget after cues in Experiment I significantly reduced RT relative to remember cues, this reduction was smaller than we had expected. Experiment II was conducted to determine whether the effectiveness of the forget after cue would be enhanced by introducing an interval between the presentation of the last pair of each list and the presentation of the cue. 
The interval provides an opportunity for processing that may lead to strengthening of set differentiation. Although segregation into sets does not insure selective search, it is plainly impossible to search selectively without set differentiation. Accordingly, a $5 \cdot \mathrm{sec}$ retention interval was introduced on each trial in Experiment II.

\section{Method}

Subjects. There were 27 subjects from the same population that provided subjects for Experiment $I$.

Material and Apparatus. The same lists and the same apparatus were used.

Procedure. The procedure of Experiment I was followed with two changes: (1) Only 12 practice trials were provided instead of the 24 trials of Experiment I. (2) Instead of presenting the remember and forget after cues immediately after the fourth pair, as was the procedure in Experiment I, a 5-sec interval was introduced between the offset of the fourth pair and the onset of the cue. The probe test immediately followed the cue. Since the cue was presented for $.5 \mathrm{sec}$, this change introduced a $5.5-\mathrm{sec}$ retention interval. This interval was also included on the forget before trials by delaying the appearance of the probe pair for $5.5 \mathrm{sec}$. The subjects were instructed to use the 5 -sec interval to organize the list for better test performance.

\section{Results}

Table 1 shows the principal results. Again RT was affected by cue, $F(2,52)=32.98$ and by solution type, $F(2,52)=10.42, p<.01$. The effects were in the same direction as Experiment 1 . Position of the probe also affected RT, $F(3,63)=26.38, \mathrm{p}<.01$, as shown in Figure 1 . The only significant interaction was Position by Cue, $F(6,126)=2.88, p<.05$, which is graphed in Figure 2.

Examination of individual data showed that $78 \%$ of the subjects had shorter RTs on forget after than on remember trials $(\mathrm{p}<.01)$ while $56 \%$ of the subjects showed faster RTs on forget before trials than on forget after trials. The latter split does not differ significantly

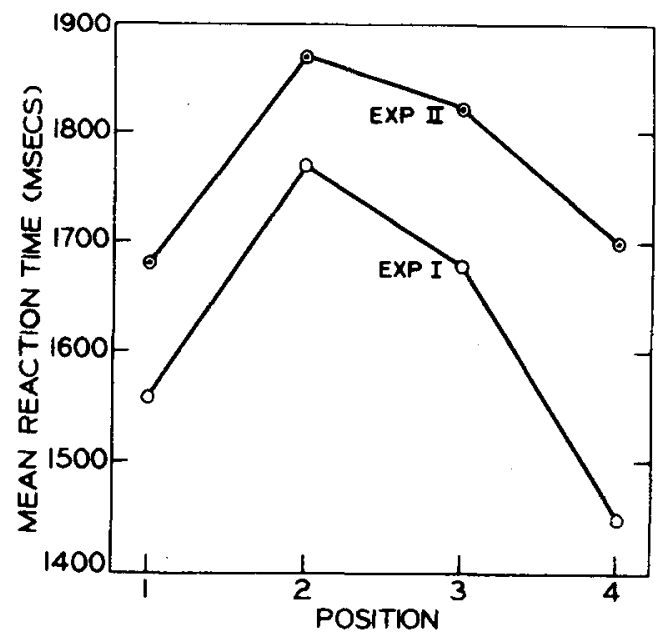

Figure 1. Serial position curves averaged over probe types and cue conditions.

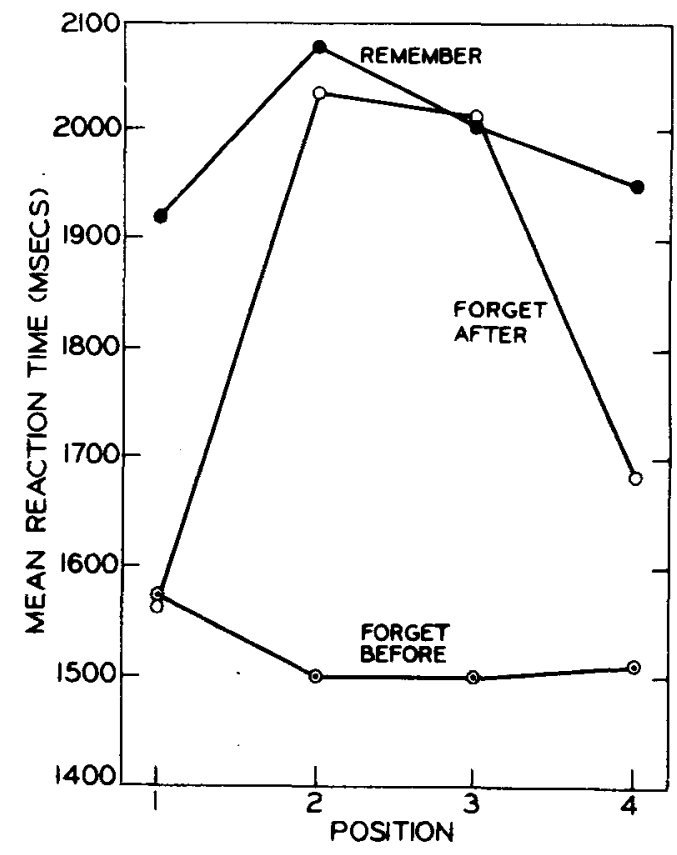

Figure 2. Cue by Position interaction in Experiment II.

from chance.

Error rate was again positively correlated with correct RT; more errors occurred on remember trials than on forget after or forget before trials.

\section{DISCUSSION}

The principal objective was to determine whether recognition would be faster on a forget trial than on a remember trial. In a sense, the forget after trial supplied the critical datum while the forget before and remember trials provided anchor points for assessing the effect of the forget after cue. If the forget after cue is fully effective in determining the size of the search set, RT on a forget after trial should be the same as RT on a forget before trial. On both of these types of trials, the search set ostensibly is limited to the cued set. On the other hand, if a forget after cue has no effect on the size of the search set, RT on a forget after trial should be the same as RT on a remember trial. The third possibility is that a forget cue provided after input does not restrict the search exclusively to the to-be-tested material, although it does reduce the search set relative to the size of the set on remember trials. In this case, RT on a forget after trial should be intermediate between RT on a remember trial and RT on a forget before trial. This, in fact, was the result in both of the current experiments. For all three solution types, remember RTs were slowest, forget before RTs were fastest, and forget after RTs were intermediate. On the assumption that RT reflects the size of the search set, this result is evidence that a forget cue provided after input will limit the size of the search set. 
Limiting the size of the search set not only decreases search time, but increases the probability of a correct recognition decision. Enhanced performance following a restricted search is a presumed benefit of adopting a selective search strategy. However, additional assumptions would be required to justify using the results of the present experiments to infer that the enhancement of recall in directed forgetting is due to restriction of the search set. In any event, the current findings do nothing to upset the emphasis on selective search. The claim that selective search is responsible for enhanced recall when a forget cue is present rests on other evidence (Epstein, 1972; Epstein, Massaro, \& Wilder, 1972; Epstein \& Wilder, 1972). In the present studies, our aim was simply to demonstrate that forget instructions can modify the size of the search set.

The results of Experiment II exhibited a significant interaction between cue and position. Ordinarily this interaction could be taken as a basis for inferences concerning the nature of the search process. However, in this case, such reasoning would be risky. The interaction is not reliable; it was not observed in Experiment I, and in our earlier work we have noted a variety of different Cue by Position interactions. Accordingly, we have not wished to rest any of our arguments on the relationship between these two variables.

The effect of the 5-sec interval in Experiment II and the instruction to organize did seem to facilitate the utilization of the forget after cue. In Experiment I, 88\% of the subjects showed faster RTs on forget before compared to forget after trials, but in Experiment II only $56 \%$ of the subjects were faster on the forget before than on the forget after trials. It is plain from Table 1 and Figure 1 that interpolation of the 5 -sec interval also had the more general effect of slowing RT. The subjects' remarks and our own observations of subject's behavior suggest that this was due to disruption of the state of test preparedness and reflects latency to the onset of search rather than duration of search.

The RT differences associated with solution-type were not a primary interest of our study. Postulating a self-terminating search would account for the faster "yes" RTs. The need to distinguish between an incorrect pair which is categorically a "yes" pair and a correct pair can account for the fact that RT for "no-same" probes was slowest.

The last question which should be considered is the fact that the forget after cue did not control the size of the search set completely. Examination of the experiments by DeRosa et al. (DeRosa, 1969; Crain \& DeRosa, 1974; Kaminsky \& DeRosa, 1972) and Howard (1973) reveals a number of instances of perfect control as well as instances of only partial control. In one respect, partial control by a postinput cue is not surprising. Selectivity probably occurs at every point in the sequence of information processing which commences with sensory reception and culminates in memory search and retrieval. However, the efficiency of the selective process may depend on the stage where it is applied. The general rule may be that the earlier selection is attempted the greater its success. In part, this may be due to the availability of easily controlled mechanisms for achieving the purpose in the early stages. No other voluntary processes that are possible in later stages rival the simple act of closing the eyes or directing the gaze as means of exercising selection. In part, selectivity may be more difficult because each additional stage of processing serves to establish or reinstate interrelationships among items which insure that if one item comes to mind others will accompany it. The answer to this question awaits more substantive studies of memory search. It is doubtful that RT data alone can provide the information we need. Perhaps, a technique of the general nature of the one employed by Anders (1971) will be more informative. Anders used a retrospective-report method to secure data on the number and location of the items searched as well as the order of search. Such data supplemented by RT data provide a more detailed picture of search than is provided by sole reliance on RT data.

\section{REFERENCES}

Anders, T. R. Retrospective reports of retrieval from short-term memory. Joumal of Experimental Psychology, 1971, 90, 251-257.

Bjork, R. A. Theoretical implications of directed forgetting. In A. W. Melton and E. (Martin (Eds.), Coding processes in human memory. New York: Winston, 1972.

Crain, R. D., \& DeRosa, D. V. Retrieval of information from multiple ensembles in short-term memory. Memory \& Cognition, 1974, 2, 255-260.

DeRosa, D. V. Transformations on sets in short-term memory: set size reduction by deletion. Joumal of Experimental Psy chology, 1969, 82, 415-426.

Epstein, W. Mechanisms of directed forgetting. In G. H. Bower (Ed.) The poychology of learning and motivation. Vol. 6. New i ork: Academic Press, 1972.

Epstein, W., Massaro, D. W., \& Wilder, L. Selective search in directed forgetting. Journal of Experimental Psychology, $1972,74,18-24$.

Epstein, W., \& Wilder, L. Searching for to-be-forgotten material in a directed forgetting task. Journal of Experimental Psychology, 1972, 95, 349-357.

Kaminsky, W., \& DeRosa, D. The influence of retrieval cues and set organization on short-term recognition memory. Joumal of Experimental Psychology, 1972, 96, 449-454.

Shebilske, W., \& Epstein, W. Effect of forget instructions with and without the conditions for selective search. Memory \& Cognition, 1973, 1, 261-267.

\section{NOTE}

1. Howard, D. V. control over memorial processing: A developmental study. Studies in attention and learning. No. 7. October, 1973. Brown University.

(Received for publication July 30, 1974; revision accepted October 12, 1974.) 